\title{
EFFET CHRONOLOGIQUE D'UNE MODIFICATION DU NIVEAU ALIMENTAIRE CHEZ LA TRUIE GESTANTE
}

\author{
E. SALMON-ITEGAGNLLR \\ avec la collaloration technique de J. RetTagiLati \\ Station de Recherches sur TÉlevage des Porcins, \\ Centre national de Recherches zootechniques, Jouy-en-Josas (Seine-ct-Oise)
}

SOMMAIRE

48 truies Large II hite, réparties en + lots (dont I lot de témoins non gestantes), ont reçu pendant la gestation une même quintité totale de nourriture, mais avec une répartition chronologique différente au cours de la gestation. On réalisait ainsi pour chaque lot, suivant le stade de gestation, les niveaux alimentaires successifs suivants : lots I et II moyen-moyen, lot III Iaut-Bas, lot IV Bas-llaut.

On observe peu de différence dans les gains de poids des truies des différents lots au cours de la gestation, à l'exception du lot ténoin non gestant, où le gain est nettement plus faible. Mais, on note que la lipogenèse est un peu plus importante dans le lot Haut-Bas et que le poids des produits de la conception est légèrement plus élevé dans le lot Bas-Haut. Le lot moyen présente des résultats intermédiaires.

Aucun des différents régimes ne paraît exercer d'effet particulier sur la lactation.

\section{INTRODUCTION}

On sait que la truie gravide est capable, pour un niveau à peine supérieur à l'entretien, de tirer un bénéfice pondéral propre de son régime alimentaire ( $\mathrm{E}$. SAIMON-LEGAGNEUR et R. JACQUOT, $196 \mathrm{I} b$ ). Cet anabolisme gravidique peut devenir plus important lorsque le niveau alimentaire est élevé et il est à l'origine de la pratique connue de la suralimentation des femelles en cours de gestation. Mais le moment auquel ce changement de niveau alimentaire risque d'être le plus efficace peut prêter à discussion. La pratique la plus courante consiste à donner un niveau alimentaire plus élevé en fin de gestation : c'est en effet à cette époque que les gains de poids de la truie gestante paraissent les plus élevés et LENKEIT et al. (I956), ainsi que RombauTs ( 1962 ), ont montré que pendant cette période il y avait notamment une rétention d'azote accrue.

Mais il n'est pas certain que ces phénomènes aient pour cause un bénéfice maternel plus important, car c'est également à cette époque que les besoins du fœtus se font le plus sentir et viennent concurrencer très sérieusement ceux propres de la mère. 
Par contre, nous avons pu constater que le phénomène d'anabolisme gravidique, qui est très précoce chez la Truie, était seul affecté par un supplément alimentaire distribué dès le début de la gestation (E. SAIMON-LEGAGxEUR et R. JACQLOT, I96I b). Il y a donc lieu de penser que les différents effets d'un changement de niveau alimentaire chez la gestante peuvent varier suivant le moment où survient cette modification.

Nous avons voulu, dans la présente expérience, essayer de préciser ce point en réalisant chronologiquement différents niveaux alimentaires au cours de la gestation chez des truies recevant un même rapport alimentaire global.

\section{MATÉRIEL E'T MÉTHODES}

48 truies adultes de race Large Il'hite appartenant i notre troupeau expérimental furent utilisées après une période de mise en condition de 3 semaines ( c cycle sexuel) depuis leur précédente lactation. Ces truies étaient réparties en 4 lots égaux en tenant compte du poids individuel, des antécédents et de l'adiposité de chaque animal. Iets truies de 3 des lots étaient alors fécondécs et celles du $4^{\mathrm{e}}$ lot servaient de témoins non grestantes.

Pendant la gestation, ou la période correspondante pour les témoins, les truies de chaque lot étaient nourries individuellement ì l'aide d'un aliment complet équilibré contenant ro p. 100 de matières azotées totales (tabl. I). Ja ration moyenne allouée pour l'ensemble de la gestation était d'un niveau supérieur de 15 p. 100 à celui des besoins d'entretien (déteminés expérimentalement lors d'une expérience précédente : ces besoins sont couverts, en moyeme, par o,9 kg d'aliment pour $100 \mathrm{~kg}$ de poids vif). Le lot témoin et l'un des lots de gestantes receraient cette même ration tout au long de la gestation, mais pour les deux aut res lots, on faisait alterner deux périodes (correspondant chacune a la moitié de la gestation) pendant lesquelles des niveaux bas (entretien), ou haut (entretien majoré de 30 p. 100) étaient appliqués.

TABLEAU I

Composition des alimenls p. Ioo

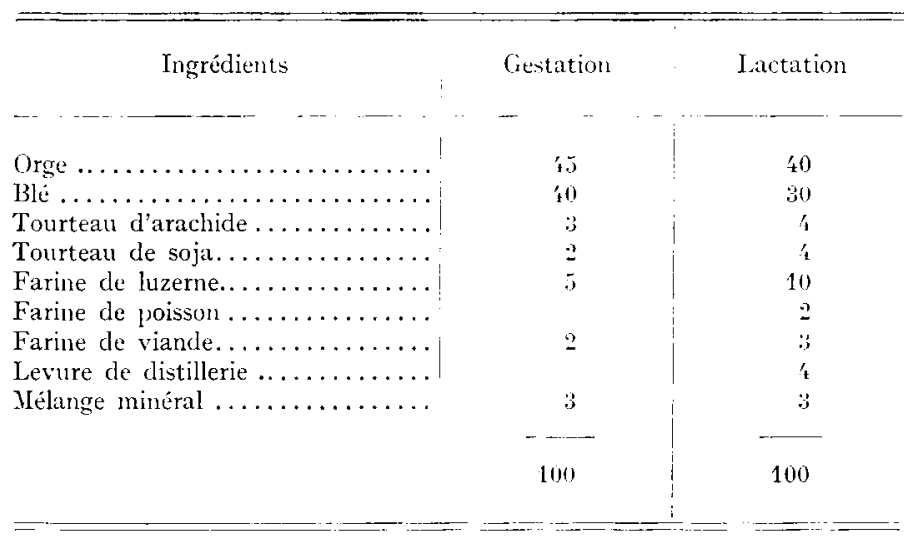

Pendant la lactation, les truies recevaient à volonté un aliment complet contenant i 6 p. ioo de matières azotées (tabl. I). Au total les truies des 4 lots recevaient donc la même quantité d'aliment. l.e schéma de l'expérience peut se résumer ainsi :

\begin{tabular}{|c|c|c|c|c|}
\hline Lot & $\begin{array}{c}\text { Rationnement } \\
-\end{array}$ & 1ere partie gestation & - parlie gestation & $\begin{array}{l}\text { Lactation } \\
-\end{array}$ \\
\hline $\mathrm{T}$ & Témoin & Entretien $+15 \mathrm{p} .100$ & Intretien $+15 \mathrm{p} \cdot 100$ & \\
\hline C.... & Constant & Entretien $+15 \mathrm{p} .100$ & Lintretien p. $15 \mathrm{p} .100$ & ad libitum \\
\hline $\mathrm{H}-\mathrm{IB}$. & Ilaut-Bas & Entrutien $+30 p \cdot 100$ & Entretien & ad libitum \\
\hline B-H... & Bas-IIaut & Entretien & Entretien $\div 30 \mathrm{p} \cdot 100$ & ad libitum \\
\hline
\end{tabular}


Pendant toute la durée de l'expérience, chaque truie ćtait pesée une fois par semaine et l'importance de ses dépôts adipeux sous-cutanés dorso-lombaires était appréciée aux ultra-sons.

A la parturition, on notait les différents renseignements concernant limportance des portées et la croissance des porcelets était suivie pendant la lactation.

\section{RÉSULTATS}

a) Variation du poids des truies

Les principaux résultats concernant le poids des truies aux différentes périodes: sont rapportés dans le tableau 2. La figure $\mathbf{I}$ indique, en outre, l'évolution du poids total au cours de ces périodes.

TABLEAU 2

Variation de poids des truies

\begin{tabular}{|c|c|c|c|c|c|c|c|c|c|c|}
\hline \multirow{3}{*}{ Lots } & \multirow{3}{*}{$\begin{array}{c}\text { Poids à } \\
\text { l'accoul- } \\
\text { plenient } \\
\text { (kg) }\end{array}$} & \multirow{3}{*}{\begin{tabular}{|} 
Poids à \\
fo j de \\
gestation \\
$(\mathrm{kg})$
\end{tabular}} & \multirow{3}{*}{$\begin{array}{c}\text { Poids à } \\
\text { la fin de } \\
\text { gestation } \\
(\mathrm{kg})\end{array}$} & \multirow{3}{*}{$\begin{array}{c}\text { Poids } \\
\text { ayrees } \\
\text { partu- } \\
\text { rition } \\
(\mathrm{kg})\end{array}$} & \multirow{3}{*}{$\begin{array}{c}\text { Poids } \\
\text { au } \\
\text { sevrage } \\
(\mathrm{kg} g)\end{array}$} & \multicolumn{5}{|c|}{ Variation de poids } \\
\hline & & & & & & Gain & total gest & ation & Gain & \\
\hline & & & & & & $0-6,0 \mathrm{j}$ & $60-115 \mathrm{j}$ & Total & gestation & lactation \\
\hline & & & & & & p. 100 & p. 100 & p. 100 & p. 100 & p. 100 \\
\hline & 206 & $2 \geq 0$ & 234 & $233^{\prime}$ & — & 7 & 7 & 14 & $1:$ & - \\
\hline C... & 207 & 233 & 267 & 247 & 236 & 12 & 16 & 28 & $1 !$ & 5 \\
\hline II-B.... & 206 & 237 & 266 & 249 & $24: 3$ & 15 & $1^{\prime}{ }^{\prime}$ & 29 & 21 & 3 \\
\hline B.H... & 205 & 231 & 268 & $2 \mathbf{\prime}^{\prime} \mathrm{t}$ & 236 & 13 & 18 & 31 & 19 & 14 \\
\hline
\end{tabular}

(1) Gain net := poids après parturition -... poids à l'accouplement.

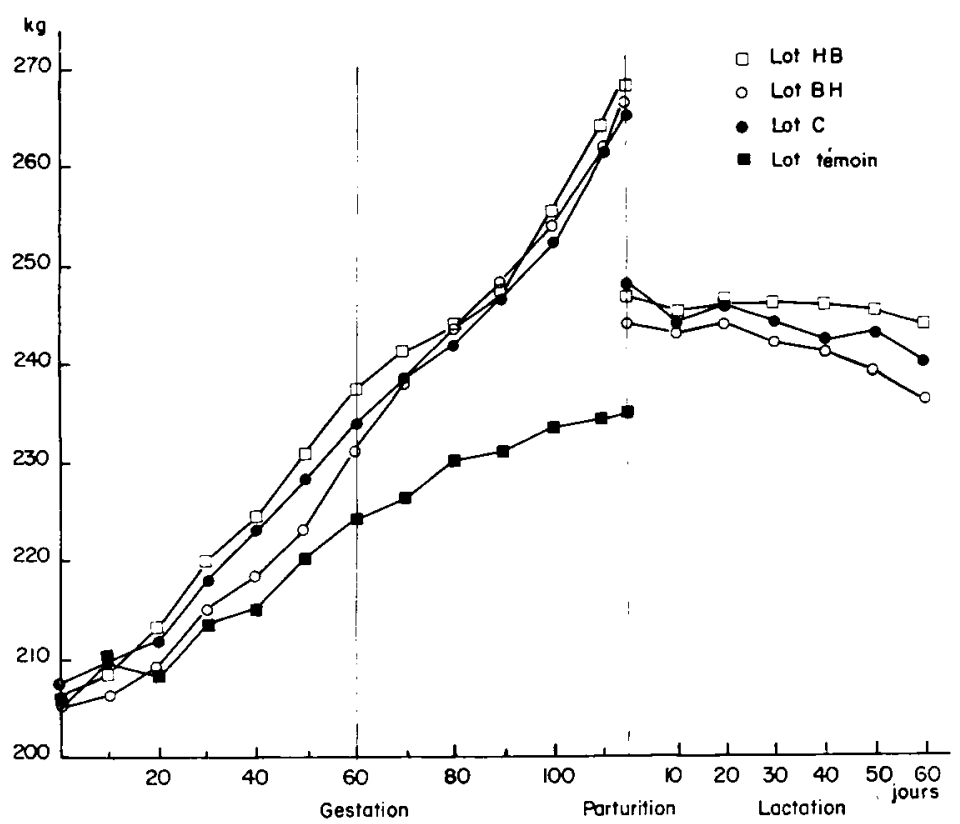

FIG. I. - Énolution du poids aif des truies 
Le résultat le plus marquant concerne la différence de comportement des témoins et des truies gestantes, qui toutes réalisent des gains de poids plus élevés pour un même apport alimentaire. On trouve ici une nouvelle confirmation de la réalité de l'anabolisme gravidique. Par ailleurs, les différences entre les gains de poids des 3 lots de gestantes sont peu importantes et en aucun cas significatives. Tout au plus peut-on parler ici de tendances.

On remarque en effet que le gain total du lot $\mathrm{B}-\mathrm{H}$ (produits de la conception compris) est peut-être le plus élevé, mais qu'il n'en est plus ainsi lorsque l'on compare les gains nets, ou bénéfices propres de la mère : c'est alors le lot $\mathrm{H}$-B quil'emporte très légèrement.

Une autre différence apparaît dans la façon dont sont réalisés les gains de poids : chez le lot témoin, les deux périodes donnent lieu à des gains identiques. Chez les gestantes, le gain est plus élevé pendant la deuxième période, sauf s'il y a restriction pendant celle-ci, comme pour le lot $\mathrm{H}-\mathrm{B}$, qui se comporte alors comme le lot témoin. On peut noter enfin que, pour chaque période, les gains de poids se classent suivant le niveau du régime.

\section{b) Tissu adipeux sous-cutané}

L'épaisseur du tissu adipeux sous-cutané constitue chez le porc un bon indice de l'adiposité totale (Dumont, I958). Ses variations renseignant donc sur l'importance de la lipogenèse pendant la période considérée.

Le tableau 3 et la figure 2 rapportent en pourcentage les variations observées chez les Truies.

TABIEAU 3

Variation du tissu adipeux sous-cutané dorsal en $p .100$ te la raleur à l'accouplement

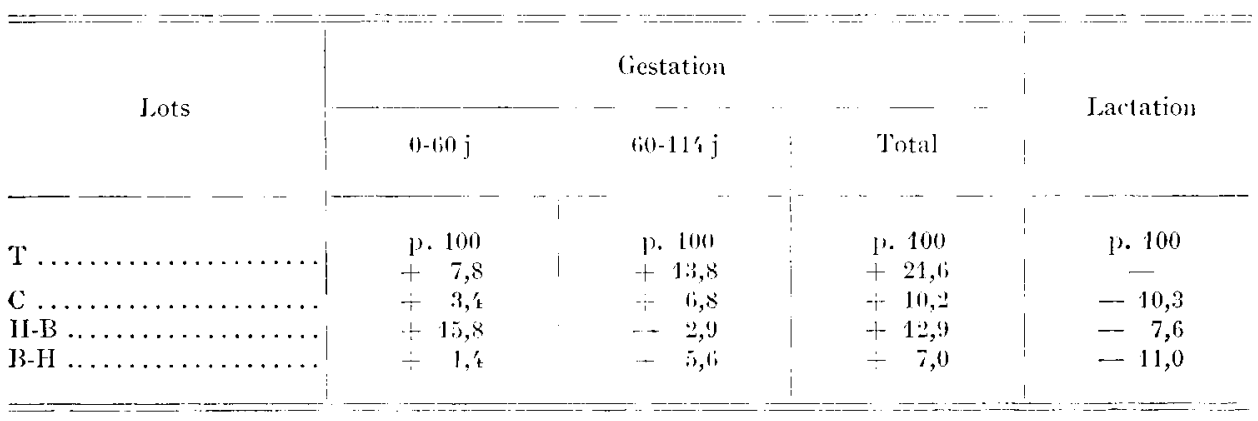

On constate que, pour l'ensemble de la gestation, la lipogenèse a été plus active chez les témoins que chez les gestantes et que, parmi ces dernières, celles du lot $\mathrm{H}-\mathrm{B}$ ont réalisé les gains les plus élevés (différence significative à $\mathrm{P}=0,05$ ), devançant dans l'ordre le lot $\mathrm{C}$ et le lot $\mathrm{B}-\mathrm{H}$. On remarque aussi que l'effet d'un même niveau alimentaire varie nettement suivant la période de distribution : par exemple, le niveau haut a provoqué une lipogenèse importante au clébut de la gestation (lot $\mathrm{H}-\mathrm{B}$ ), mais plus faible en fin de gestation (lot $\mathrm{B}-\mathrm{H}$ ). De même, le niveau bas permet une légère augmentation du tissu adipeux en début de gestation (lot $\mathrm{B}-\mathrm{H}$ ) et provoque une diminution en fin de gestation (lot $\mathrm{H}-\mathrm{B}$ ). La figure 2 montre que dans ce cas, 
cette mobilisation des lipides a surtout lieu pendant les toutes dernières semaines de la gestation.

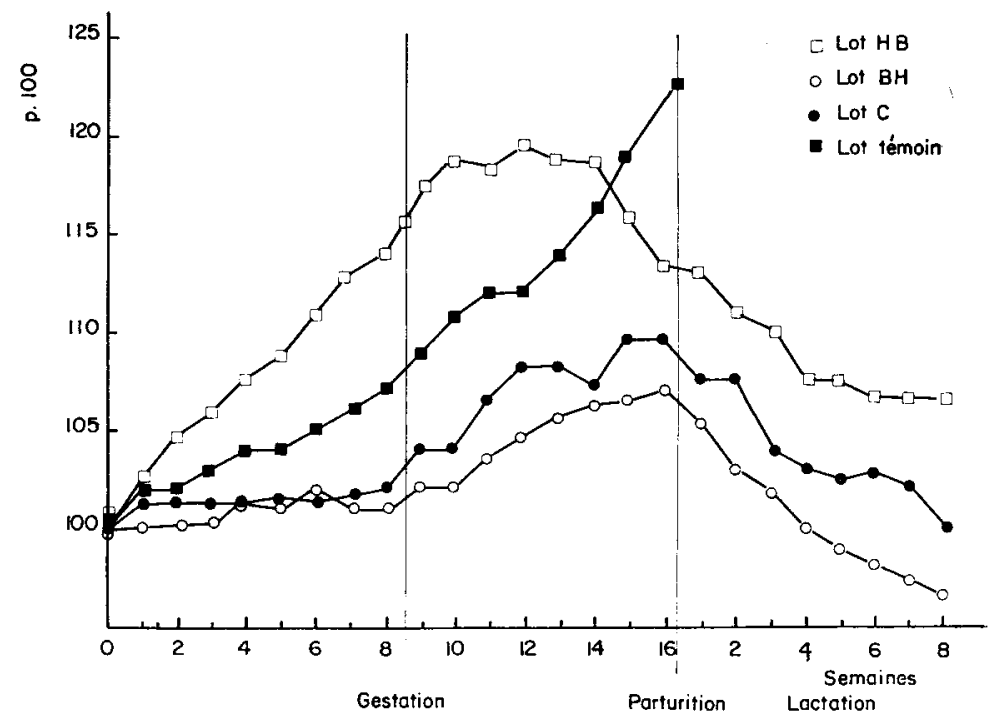

FIG. 2. - Évolution du tissu adipeux sous-cutané dorsal (p. roo des valeurs à l'accouplement)

\section{c) Parturition}

Les différentes caractéristiques des portées à la naissance sont rapportées au tableau 4 .

TABLEAU 4

Répartition des différents produits de la conception à la parturition

\begin{tabular}{|c|c|c|c|c|c|c|c|c|}
\hline \multirow[b]{2}{*}{ Lots } & \multicolumn{4}{|c|}{ Poids des productions } & \multicolumn{3}{|c|}{ Porcelets } & \multirow[b]{2}{*}{$\begin{array}{l}\text { Durée de la } \\
\text { parturition }\end{array}$} \\
\hline & $\begin{array}{c}\text { Porcelets } \\
(\mathrm{kg})\end{array}$ & $\begin{array}{l}\text { Placenta } \\
(\mathrm{kg})\end{array}$ & $\begin{array}{l}\text { Liquides } \\
\text { (kg) }\end{array}$ & $\begin{array}{l}\text { Total } \\
(\mathrm{kg})\end{array}$ & $\begin{array}{l}\text { Nombre } \\
\text { vivants }\end{array}$ & $\begin{array}{l}\text { Nombre } \\
\text { morts }\end{array}$ & $\begin{array}{l}\text { Poids } \\
\text { moyen } \\
\text { des } \\
\text { vivants } \\
\text { (kg) }\end{array}$ & \\
\hline$C \ldots \ldots \ldots \ldots$ & 14,5 & 2,6 & 3,3 & 20,4 & 10,1 & 0,8 & 1,40 & $4 \mathrm{~h} 40 \mathrm{mn}$ \\
\hline $\mathrm{H}-\mathrm{B} \ldots \ldots \ldots \ldots$ & 13,4 & 2,1 & 2,9 & 18,6 & 10,1 & 1,1 & 1,26 & $4 \mathrm{~h} 42 \mathrm{mn}$ \\
\hline B-H.......... & 15,6 & 2,9 & 4,8 & 23,3 & 10,6 & 1,5 & 1,38 & 5 h $24 \mathrm{mn}$ \\
\hline
\end{tabular}

On ne note pas de différence significative entre les différents lots. On peut remarquer toutefois que chez le lot $\mathrm{B}-\mathrm{H}$, l'ensemble des produits de la conception paraît plus important que chez les lots $\mathrm{C}$ et $\mathrm{H}-\mathrm{B}$, dans l'ordre. Cette différence paraît provenir du poids total des foetus, légèrement plus nombreux, et surtout 
du poids des différentes pertes liquides au moment de la parturition. Un niveau alimentaire élevé en fin de gestation semble donc favoriser légèrement la croissance des foetus. A noter que dans ce cas, la durée de la parturition est également un peu plus élevée.

\section{d) Lactation}

Les performances de lactation ont été sensiblement les mêmes dans les trois groupes, comme le montre le tableau 5. Aucune différence n'est significative.

TABLEAU 5

Lactations

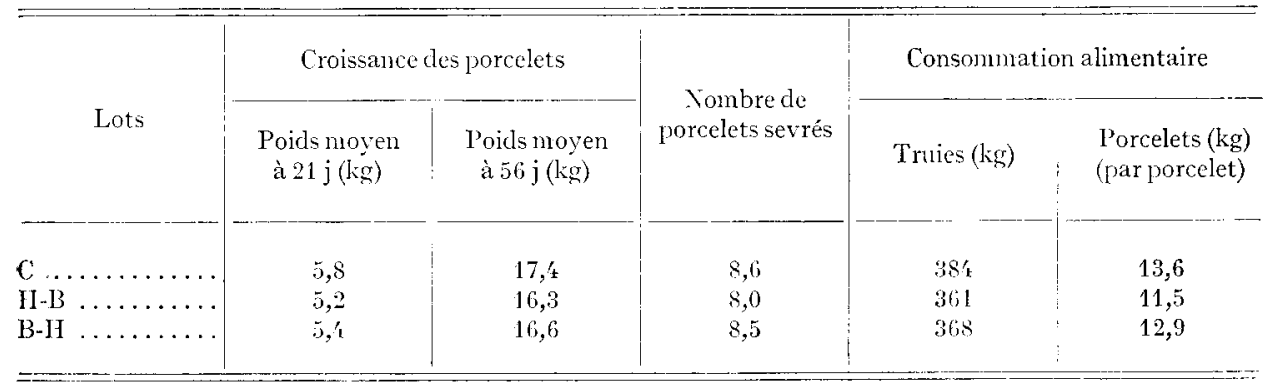

\section{DISCUSSION}

Les gains de poids totaux (gain net + produits de la conception) réalisés au cours de la gestation ont pratiquement été les mêmes dans les trois groupes expérimentaux. On ne peut donc dire, au vu de ce seul aspect, que l'un des régimes se soit montré supérieur à l'autre.

On peut remarquer toutefois que les gains de poids ont été en général plus élevés pendant la seconde moitié de la gestation que pendant la première, ce qui, comparé à ce qui se passe chez les témoins non gestantes, paraît être une caractéristique de l'état de gestation. Dans ce domaine le lot $\mathrm{H}-\mathrm{B}$ a réalisé les gains les plus élevés. Mais il est en particulier remarquable que le lot $\mathrm{B}-\mathrm{H}$, relativement sousalimenté pendant la deuxième partie de la gestation, ait continué à prendre du poids à un rythme supérieur aux témoins, ce qui, même après déduction des produits de la conception, laisse un bénéfice net non négligeable pour cette période.

L'ensemble de ces considérations rejoint done les conclusions de Rombauts (Ig62) sur l'augmentation de la rétention azotée en fin de gestation. Toutefois, le fait qu'une partie de la différence entre les résultats des régimes $\mathrm{H}-\mathrm{B}$ et $\mathrm{B}-\mathrm{H}$ paraisse provenir du poids des produits de la conception (tab1. 4), montre bien que le supplément alimentaire en fin de gestation a profité en priorité, dans des limites qui restent à préciser, à ces productions et notamment aux fœtus. Ceci apparaissait déjà dans les expériences de MíTcheli, et al. (r93I) et se trouve en parfait accord avec les résultats obtenus, avec des régimes croisés, par WAIIACE (I948) chez la 
Brebis. Il en découle qu'en ce qui concerne l'anabolisme gravidique vrai de la Truie, un supplément alimentaire ne paraît pas plus efficace en fin de gestation qu'au début. C'est ce que confirme le résultat trouvé pour le gain net des 'Truies après parturition : celui-ci est même légèrement moins élevé dans le cas du régime $B-H$ que du régime $\mathrm{H}-\mathrm{B}$.

Il est toutefois inexact de penser que les gains de poids réalisés dans ces conditions aient la mème valeur. Dans le cas du lot $\mathrm{H}-\mathrm{B}$, en effet, la quantité de tissu adipeux élaboré paraît nettement plus importante et ceci est dû essentiellement à l'effet du régime haut au début de la gestation. Si l'on compare les résultats obtenus par les gestantes et les témoins, on constate qu'un même supplément alimentaire (niveau $\mathrm{H}$ ) provoque chez les gestantes une lipogenèse supérieure à celle des témoins au début de la gestation et inférieure en fin de gestation. On obtient le même classement lorsqu'on compare l'effet d'un niveau bas. Ceci montre très nettement que l'aptitude à stocker de l'énergie sous forme de tissu adipeux est plus élevée au début qu'à la fin de la gestation. Ceci peut encore s'expliquer par les besoins provoqués par l'édification des foctus : au début de la gestation toute l'énergie du régime est disponible au bénéfice cle la gestante, qui se comporte alors comme un animal en fin de croissance et construit des tissus de plus en plus riches en graisse. En fin de gestation, il y a compétition entre les besoins du fuetus et ceux de la mère ; la fraction d'énergie conservée par celle-ci devient beaucoup plus faible et, si le régime est faible ou insuffisant (lot $\mathrm{H}-\mathrm{B}$ ), il peut y avoir mobilisation des dépôts adipeux, phénomènes que nous avions déjà constatés lors de précédentes expériences (SALMONLEGaGNEUR et JACQUoT, Ig6I a). Il est alors paradoxal de voir, qu'en dépit de cette carence énergétique, la 'Truie peut toutefois continuer à anaboliser à son profit ; mais dans ce cas, il ne peut plus s'agir que de tissu protéique, moins cồteux à élaborer.

On en vient ainsi à penser que si l'anabolisme azoté peut se poursuivre d'une façon régulière au profit de la mère tout au long de la gestation, même lorsque les conditions alimentaires sont défavorables, l'anabolisme des tissus lipidiques n'a lieu en fin de gestation que si la ration est abondante. Ceci revient à donner à l'énergie de la ration un rôle de facteur limitant dans les phénomènes d'anabolisme gravidique, hypothèse qu'avait déjà formulée, par d'autres roies, PIKE (I945) sur la Ratte.

In ce qui concerne la lactation, les légères différences observées dans la ritesse de croissance des porcelets semblent relever davantage des différences de consommation cles truies et des porcelets au cours de la lactation, que de l'effet du régime de gestation. Il en va de même des pertes de poids des truies pendant la lactation.

En conclusion, les cleux régimes $\mathrm{H}-\mathrm{B}$ et $\mathrm{B}-\mathrm{H}$ de notre expérience se distinguent essentiellement par un accroissement cles réserves lipidiques de la mère pour le premier et par une légère augmentation du poids des produits de la conception pour le second. Comme on pouvait le penser, le régime C, ou moyen, a cles effets intermédiaires. Suivant le but recherché, l'un ou l'autre de ces régimes pourrait donc être préconisé. Toutefois, ces effets restent faibles et aucune influence immédiate sur la lactation n'a pu être décelée. Aucune de ces différentes solutions ne paraît donc s'imposer; la plus simple parait la plus indiquée. 


\title{
SUMMARY
}

\author{
CHRONOLOGIC EFFECT OF CHANGE IN PLANE OF NUTRITION IN PREGNANT SOWS
}

An experiment was made on 3 groups of 12 pregnant sows and one group of controls to study the effect of chronologic change in plane of feeding during pregnancy. Total intake was the same in all groups, but pregnancy was divided in two equal periods with the following successive rations for each group of sows. I medium-medium (controls), II medium-medium (pregnant), III high-low (pregnant), IV low-high (pregnant).

The results are as follows :

r. There is no significant difference in total and net gain in weight between sows of all groups, except controls (total gains were respectively $(\mathrm{kg}): 28-60-60-63$ ).

2. Products of conception are slightly more heavy in group IV and, after control group, lipogenesis is more important in group III. This may indicate a change in partition of nutrients among sows and litters in the course of pregnancy.

3. No definite influence is found on lactation results. interest.

It is concluded that overfeeding sows at either part of pregnancy seems of small practical

\section{RÉFÉRENCES BIBLIOGRAPHIQUES}

DUMONT 3. I.., I958. Méthodes indirectes de mesure de la graisse corporelle des mammifères. Cahiers techniques CNCERNA, 2, 95-I 58 .

Lenkeir W., Gutte J. O., Kirchioff W., Soehngen F. K., Farries E., i 956 . Weitere untersuchungen zur Abhängigeit des N-Umsatzis während der Laktation von der Nährstoffversorgung Während der Gravidität. Ztschr. Tierern. F'uttermittelk., 11, 337-352.

Mitchell H. II., Carroll W. E., HamltoN 'T.S., IIU.NT G. E., i93I. Food requirements of pregnancy in swine. III. Agr. Exp. Sla. Bull., 375, 467-504.

Pike R. I., Suder H. B., Ross M. L., I954. The influence of protein and energy intakes upon nitrogen retention in pregnant rats. J. Nutr., 52, 297-309.

Ronbauts P., I962. Évolution de l'anabolisme gravidique chez la Truie en fonction de l'âge de l'animal. Ann. Zootech., 11, 39-5I.

Salmon-Legagneur E., JaCQuor R., I96I a. Modifications corporelles entraînées par l'anabolisme gravidique chez la Truie. C. R. Acad. Sci., 253, 544-546.

SaLMON-Legagneur E., JaCQuot R., ig6 i $b$. Influence du niveau alimentaire sur le comportement nutritionnel de la Truie gestante. C. R. Acad. Sci., 253, I497-1499.

WALLACE L. R., I948. The growth of lambs before and after birth in relation to the level of nutrition. $J$. Agr. Sci., 38, $367-402$. 